Revue musicale OICRM

\title{
Reconstruire les numéros chantés du Verfügbar aux Enfers
}

\section{Le cas de l'air de Rosine}

\section{Christophe Gauthier}

Volume 3, numéro 2, 2016

Mémoire musicale et résistance. Autour du Verfügbar aux Enfers de Germaine Tillion

URI : https://id.erudit.org/iderudit/1060109ar

DOI : https://doi.org/10.7202/1060109ar

Aller au sommaire du numéro

Éditeur(s)

OICRM

ISSN

2368-7061 (numérique)

Découvrir la revue

Citer cet article

Gauthier, C. (2016). Reconstruire les numéros chantés du Verfügbar aux

Enfers : le cas de l'air de Rosine. Revue musicale OICRM, 3(2), 99-116.

https://doi.org/10.7202/1060109ar
Résumé de l'article

La nature même de l'opérette-revue Le Verfügbar aux Enfers, œuvre collective et orale, pose de nombreux défis à sa restitution musicale et à sa performance. Le présent article se penche sur ces défis, dont le premier est de déterminer si l'on cherchera à reproduire ce que les déportées de Ravensbrück avaient les moyens de faire à l'intérieur du camp, ou plutôt à reconstruire et réinterpréter ce qu'elles avaient en tête. L'article avance des pistes de réponses à ces questions, et propose une reconstruction originale d'un air du Verfügbar aux Enfers basé sur un duo de l'opérette Ciboulette (1923) de Reynaldo Hahn (1874-1947) 


\title{
Reconstruire les numéros chantés du Verfügbar aux Enfers. Le cas de l'air de Rosine \\ Christophe Gauthier
}

\begin{abstract}
Résumé
La nature même de l'opérette-revue Le Verfügbar aux Enfers, œuvre collective et orale, pose de nombreux défis à sa restitution musicale et à sa performance. Le présent article se penche sur ces défis, dont le premier est de déterminer si l'on cherchera à reproduire ce que les déportées de Ravensbrück avaient les moyens de faire à l'intérieur du camp, ou plutôt à reconstruire et réinterpréter ce qu'elles avaient en tête. L'article avance des pistes de réponses à ces questions, et propose une reconstruction originale d'un air du Verfügbar aux Enfers basé sur un duo de l'opérette Ciboulette (1923) de Reynaldo Hahn (1874-1947).
\end{abstract}

Mots clés : Reynaldo Hahn ; Le Verfügbar aux Enfers ; performance ; reconstruction ; Germaine Tillion.

\begin{abstract}
By its very nature as a collective and oral work, the operetta-revue Le Verfügbar aux Enfers poses many challenges for musical restoration and performance. This article examines these challenges, the first of which questions whether we should reproduce what the Ravensbrück deportees could actually have done with the means they had within the camp, or whether we should try to reconstruct and reinterpret the work according to their intentions. The article suggests some answers to these questions, and proposes an original reconstruction of an air from Le Verfügbar aux Enfers based on a duet from the operetta Ciboulette (1923) by Reynaldo Hahn (1874-1947).
\end{abstract}

Keywords: Reynaldo Hahn ; Le Verfügbar aux Enfers ; performance ; reconstruction ; Germaine Tillion.

En avril 1945, Germaine Tillion était évacuée du camp de concentration de Ravensbrück, emportant avec elle, grâce à l'aide d'une camarade ${ }^{1}$, une opéretterevue : Le Verfügbar aux Enfers. Euvre collective et orale, franchement drôle, mais tout

1 C'est en effet Jacqueline d'Alincourt qui a sorti le manuscrit du camp au moment de l'opération Bernadotte. Voir l'avant-propos de Tzvetan Todorov dans Tillion 2005, p. 3. 
autant crue et violente, il s'agit pour le moins d'un document curieux. Les auteures font alterner passages récités et passages chantés ; or, elles n'ont composé aucune musique, utilisant plutôt des airs et mélodies connus, auxquels elles ont associé de nouvelles paroles. On y reconnaît des airs d'opéras, de la mélodie française, et même de la musique publicitaire ${ }^{2}$.

Le présent article se penche sur les défis qu'impliquent la restitution musicale et la performance du Verfügbar aux Enfers. La nature de cette œuvre pose un défi à la performance, entre autres parce qu'il n'existe pas à proprement parler de référent permettant de déterminer si la conception et l'interprétation coïncident avec ce qui a été imaginé au départ par les auteures. Ces dernières n'ayant pas prévu de mise en scène, plusieurs choix peuvent être effectués, modifiant ainsi la réception de l'œuvre. Pour répondre aux exigences de ce défi, une proposition de reconstruction d'un air du Verfügbar aux Enfers, parodie d'un duo extrait de l'opérette Ciboulette (1923) du compositeur Reynaldo Hahn (1874-1947), sera ici présentée.

Avant d'aller plus loin dans cet exercice, il importe de préciser qu'il ne sera pas possible de statuer sur une version préférable, puisqu'il existe plusieurs options qui sont toutes aussi pertinentes les unes que les autres. Cependant, il est essentiel de choisir dès le départ la direction générale que prendra cette réinterprétation : veut-on tenter de reproduire ce que les déportées de Ravensbrück avaient les moyens de faire à l'intérieur du camp ou veut-on essayer de reconstruire et réinterpréter ce que les détenues avaient en tête?

Peu importe l'option choisie, chacune d'elle présente des limites qu'il est nécessaire d'exposer. Dans le cas où l'on choisit de reproduire ce que les prisonnières avaient les moyens de faire, jusqu'où faut-il aller? Germaine Tillion et ses compagnes d'infortune ont créé cette œuvre dans la clandestinité la plus totale et elles ont dû se contenter d'en chuchoter des passages entre deux corvées. Il n'est ni possible, ni souhaitable de recréer de telles conditions. Dans le cas où l'on voudrait reproduire ce que les détenues avaient en tête, peut-on prétendre savoir exactement ce qu'elles entendaient? Comme il sera explicité plus loin, le fait de connaître les mélodies auxquelles elles faisaient référence ne permet pas nécessairement d'avoir une idée exacte de ce qu'elles avaient en tête. Leur mémoire n'étant pas infaillible, il existe parfois des différences très importantes entre les airs d'origine et ce qu'elles semblent en avoir retenu. Aux fins de cet exercice, un amalgame de ces deux options sera préféré : sans tenter de recréer les conditions dans lesquelles les détenues créaient Le Verfügbar aux Enfers, il semble en effet souhaitable de chercher à reproduire ce qu'elles avaient les moyens de faire.

Le choix d'effectuer un amalgame de ces deux options influencera également d'autres considérations, par exemple la question de savoir si, dans une représentation scénique du Verfügbar aux Enfers, il faut miser sur l'embauche de chanteuses professionnelles ou sur celle d'actrices n'ayant pas nécessairement de formation lyrique ${ }^{3}$.

2 Sur les différents registres musicaux convoqués dans Le Verfügbar aux Enfers, voir l'article de Marie-Hélène Benoit-Otis et Philippe Despoix dans le présent numéro.

3 Ces deux possibilités ont été explorées dans les mises en scène du Verfügbar aux Enfers présentées à ce jour ; voir à ce sujet l'article de Cécile Quesney dans le présent numéro. 
Il sera aussi important de déterminer si un accompagnement instrumental sera prévu ; et si oui, quels instruments utiliser? Question plus qu'essentielle, considérant que les instruments de musique étaient prohibés à Ravensbrück. Enfin, le rôle du naturaliste sera-t-il interprété par un homme, comme semble l'avoir prévu l'opérette-revue, ou par une femme, comme les conditions du camp le permettaient?

S'il est un sujet hautement complexe, c'est bien celui du rire et du traitement humoristique dans Le Verfügbar aux Enfers. Nous ne sommes pas ici en présence d'une œuvre de fiction au sens habituel du terme, pas plus que d'une reproduction fidèle et entière de ce que pouvaient vivre ces femmes : sans être un témoignage au sens premier du terme, l'opérette-revue décrit la réalité du camp dans lequel vivaient les détenues, même si elle le fait sur un ton résolument humoristique. Ce paradoxe amène la question suivante : cet humour, qui, en plus d'être une réponse à la déshumanisation du camp nazi, répondait à un besoin de survie, peut-il faire rire aujourd'hui ? Plus concrètement, Le Verfügbar aux Enfers doit-il faire rire ou faire pleurer? On se souvient que le film à succès Schindler's List (Steven Spielberg, 1993) a été l'objet de controverses, parce que certains jugeaient qu'il présentait une vision enjolivée de la réalité concentrationnaire ${ }^{4}$. C'est également le cas du film de Roberto Benigni La vita è bella (1997) : plusieurs critiques ont reproché au réalisateur de faire rire les spectateurs avec un film abordant la Shoah. Une reconstruction comique du Verfügbar aux Enfers pourrait également s'exposer à de telles critiques.

Germaine Tillion elle-même a gardé longtemps pour elle le manuscrit, parce qu'elle craignait que les gens ne comprennent pas l'humour omniprésent dans l'opérette-revue. Elle se disait qu'on la croirait folle (Unger 2008) ${ }^{5}$. À une époque où plusieurs personnes niaient encore l'existence de l'Holocauste, elle redoutait également qu'on pense que la vie dans les camps était légère. Or, après que des survivantes du camp eurent assisté à une représentation de l'œuvre jouée de façon très comique, l'une d'elles a explicitement approuvé ce choix de mise en scène (ibid.).

\section{LA RECONSTRUCTION DES AIRS DU VERFÜGBAR AUX ENFERS}

Compte tenu des questions générales soulevées par la représentation du Verfügbar aux Enfers, l'une d'elle ressort du lot, plus concrète : celle de la mise en musique en elle-même et des écueils qui l'accompagnent. Rappelons que Germaine Tillion n'a laissé aucune musique écrite. Elle a plutôt indiqué, à la fin de la plupart des airs, la mélodie originale à laquelle chacun se rapporte. Certaines de ces mélodies sont rendues facilement reconnaissables par leur titre et ont été aisément identifiées. D'autres étaient présentées de manière moins précise et ont nécessité un certain travail pour retracer leur origine. Les éditeurs du manuscrit de Germaine Tillion (Todorov, Andrieu et al.) sont parvenus à reconnaître une grande partie des sources musicales, ainsi que les compositeurs et metteurs en scène qui ont recréé l'opérette

\footnotetext{
$4 \quad$ Voir par exemple Siroka 2012.

5 Sur la publication tardive du Verfügbaraux Enfers, voir également l'article de Marie-Hélène Benoit-Otis et Philippe Despoix dans le présent numéro.
} 
depuis 2007 (Christophe Maudot et Danielle Stéfan, entre autres). Aujourd'hui, à une seule exception près, tous les airs auxquels les auteures faisaient référence ont été jumelés à leur pièce d'origine.

N'ayant sous la main ni partition, ni enregistrement, les auteures du Verfügbar aux Enfers ont dû se fier à leur mémoire musicale au moment d'écrire de nouvelles paroles sur des airs connus. Elles ont probablement entendu certains de ces airs quelques fois seulement, ce qui ne leur a pas permis de les connaître par cœur. Pour cette raison, il peut parfois être ardu d'adapter les nouveaux textes aux musiques existantes. La prosodie n'étant pas toujours cohérente, certains vers sont trop longs et d'autres trop courts. Parfois, les auteures ont omis des sections entières d'un air ; sa reconstruction peut donc rapidement s'apparenter à un casse-tête.

Ces difficultés soulèvent un dilemme crucial : dans les cas problématiques, faut-il retravailler le texte de Germaine Tillion pour le faire correspondre à la musique originale, ou faut-il au contraire adapter la musique au texte ? La première option permet d'arriver à un résultat « fini » et artistiquement intéressant, mais elle nécessite de réorganiser et de modifier le texte du manuscrit.

Comme on l'a vu, le processus de reconstruction du Verfügbar aux Enfers implique de nombreux questionnements dont l'issue peut se présenter sous des formes variables. Or, s'il existe une donnée certaine, c'est bien le document que nous a laissé Germaine Tillion. Il s'agit en fait du seul document concret disponible. N'est-il pas périlleux de l'altérer pour faciliter l'adaptation du texte à la musique ? Pour que le résultat soit fidèle à l'œuvre collective, il nous semble plus approprié d'emprunter la seconde voie : adapter la musique originale au texte de Germaine Tillion ${ }^{6}$.

Cette tâche ne s'exécute pas aisément, surtout lorsqu'il s'agit d'airs très connus. Plutôt que de tronquer des passages du texte de Tillion, ce sont des phrases musicales, et parfois des sections entières d'un air, qu'il faut modifier. Évidemment, il restera toujours des variables inconnues, car cette option exige également de prendre des décisions. Il n'en demeure pas moins que les choix effectués s'approchent de ce que les déportées avaient en tête - ou à tout le moins, ils ne s'en éloignent pas de façon délibérée.

\section{Proposition DE RECONSTRUCTION D'UN AIR}

Afin de pousser cette démonstration jusqu'à la limite du possible, nous avons tenté d'exécuter la reconstruction d'un air du Verfügbar aux Enfers. L'air sélectionné, "Nous avons fait un beau voyage ", est l'un de ceux qui présentent le plus grand défi dans l'opérette-revue. Dans son manuscrit, Germaine Tillion a indiqué que ce passage correspond à un "Air de Ciboulette » (Tillion 2005, p. 154-155). Il s'agit en fait d'une référence au duo "Nous avons fait un beau voyage », tiré de l'opérette Ciboulette de Reynaldo Hahn.

6 Sur ce type d'approche, voir également l'article de Catherine Harrison-Boisvert et Caroline Marcoux-Gendron dans le présent numéro. 
Cette étude s'appuyant sur une démarche personnelle, il nous a fallu d'abord répondre aux questions soulevées plus haut. Étant donné que le souci principal est de recréer le plus fidèlement possible ce que les détenues avaient la possibilité de faire dans le camp, les effectifs et les accessoires seraient limités à ce qu'elles pouvaient avoir sous la main. L'œuvre serait interprétée exclusivement par des femmes, y compris le rôle du naturaliste, puisque Ravensbrück était un camp pour femmes et que les auteures n'étaient que des femmes. Nous ne ferions pas appel à des chanteuses, mais plutôt à des actrices, car les détenues n'avaient pas nécessairement une voix entraînée. Puisque les détenues ne disposaient pas d'instruments de musique, il n'y aurait aucun accompagnement instrumental. Tout au plus, on pourrait utiliser des objets tels que des casseroles en guise d'instruments percussifs ${ }^{7}$.

L'air "Nous avons fait un beau voyage " est un véritable voyage culinaire, où sont décrits de nombreux mets régionaux de la France. Il n'était pas rare pour les prisonniers des camps de noter des recettes, la nostalgie culinaire étant utilisée comme moyen de résister à la faim ${ }^{8}$. On devine que les déportées ont dû ressentir une certaine satisfaction en se rappelant les spécialités culinaires de leurs régions respectives. Et on comprend qu'elles en avaient beaucoup à dire, car il s'agit de l'air le plus long du Verfügbar aux Enfers !

Bien que l'air original soit en fait un duo, nous avons choisi d'en faire un solo, d'abord parce que Germaine Tillion indique en didascalie "Rosine (chantant) » (Tillion 2005, p. 154-155) et qu'il n'est fait mention d'aucun autre personnage. De plus, puisque Tillion fait référence au bas de la page à un "air de Ciboulette " plutôt qu'à un duo, il ne faut pas écarter la possibilité qu'elle n'ait simplement pas retenu le fait qu'il s'agit d'un duo.

Pour préserver le caractère oral de l'opérette-revue et pour tenir compte du fait qu'elle a été composée à partir de la mémoire auditive des déportées, il nous a semblé préférable d'éviter de travailler à partir de la partition originale de l'opérette Ciboulette. Si l'objectif est de se rapprocher de ce que les détenues avaient en tête, il n'est pas approprié de s'appuyer sur un support dont elles ne disposaient pas. Dans le cas du Verfügbar aux Enfers, la partition aveugle davantage qu'elle n'éclaire, parce qu'un musicien a l'habitude de se fier à une partition et qu'il a du mal à s'en détacher. Après plusieurs écoutes du duo de Ciboulette, la version enregistrée en 1923 par Edmée Favart et André Baugé est proposée en exemple. Cette version a été réalisée par les chanteurs de la distribution originale de Ciboulette ${ }^{9}$; Germaine Tillion et ses compatriotes la connaissaient probablement.

7 Les didascalies du Verfügbar aux Enfers comportent d'ailleurs plusieurs passages où des objets du quotidien du camp sont utilisés en guise d'instruments de percussion ; pour plus de détails, voir l'article de Marie-Hélène Benoit-Otis et Philippe Despoix dans le présent numéro.

8 Voir par exemple le témoignage d’Édith Combus, déportée à Ravensbrück, dans le film Festins imaginaires (Anne Georget, 2015).

9 Pour plus d'informations sur Ciboulette, voir la page qui lui est consacrée dans l'Encyclopédie multimédia de la comédie musicale théâtrale en France, 1918-1944 : http://194.254.96.55/cm/?for=fic\&cleoeuvre=75 (consulté le 10 novembre 2014). 
Une écoute répétée du duo sur une période s'échelonnant sur près d'un mois nous a permis de nous familiariser avec ce dernier. Toutefois, même après l'avoir entendu plusieurs fois, et peut-être plus souvent encore que Germaine Tillion, il demeure difficile de fredonner la mélodie de mémoire, surtout en ce qui concerne les couplets. Sans accompagnement instrumental pour les préparer, il est assez difficile de retenir les changements de tonalité.

Le duo de Ciboulette est divisé en trois sections (voir l'annexe A pour le découpage de la partition). La section $\mathrm{A}$, en si bémol majeur, module dans le ton de la dominante. La section B reprend dans le ton initial et module dans le ton de ré mineur, puis dans celui de do mineur. Cette section consiste en une énumération de tout ce qu'ont rencontré Ciboulette et Duparquet pendant leur voyage ${ }^{10}$. Enfin, le début de la section $\mathrm{C}$ rappelle rythmiquement la section $\mathrm{A}$ et commence avec les mêmes mots. Elle débute en do mineur et une marche harmonique modulante ramène au ton initial de si bémol majeur.

Tout porte à croire que les auteures du Verfügbar aux Enfers n'ont pas retenu cette forme au moment de composer l'air de Rosine. La façon dont Germaine Tillion a disposé les paragraphes laisse croire qu'elles en ont fait une chanson de forme refraincouplet (le texte est reproduit dans l'annexe C). Les quatre premiers vers correspondent à la section $\mathrm{A}$ du duo original. Les huit vers suivants, placés en retrait, sont une énumération de plats régionaux français et correspondent, du moins en partie, à la section B (pour qu'elle soit complète, il aurait fallu que les auteures ajoutent quatre vers). Enfin, le retour immédiat au refrain donne l'impression qu'elles ont omis la section $\mathrm{C}$ du duo original, la confondant sans doute avec la section A.

Ce que les auteures ont retenu de la section B est moins évident. Dans le duo de Ciboulette, on peut la diviser en trois sous-sections, dont chacune comprend quatre vers. La première ne module pas et reste dans le ton initial de si bémol majeur. La deuxième module en ré mineur et la troisième module en do mineur. Il est cependant difficile, voire impossible, de mémoriser les deux dernières sous-sections sans avoir la partition sous les yeux. Compte tenu des nombreuses modulations, il aurait été surprenant que les auteures puissent les chanter. Même si certaines d'entre elles étaient douées d'une meilleure oreille que la majorité des gens - la chanteuse Jany Sylvaire, qui a enregistré le duo après la guerre, a d'ailleurs fait partie du groupe de Germaine Tillion avant d'être transférée à Neubrandenburg -, il aurait été exceptionnel qu'elles aient pu retenir les deux premières sous-sections, car la seconde se termine en ré mineur : sans accompagnement instrumental et sans partition, il n'est pas facile de revenir au ton de si bémol majeur - ou dans un ton majeur tout court - pour le refrain.

Face à une telle situation, deux solutions sont possibles. En premier lieu, le texte du couplet pourrait être simplement déclamé, ce qui écarte les difficultés liées à la modulation. Une deuxième option consiste à ne conserver que la première sous-section de $\mathrm{B}$, qui contient quatre vers et qui ne module pas, et à la répéter une seconde fois pour

10 Sur le contexte d'apparition du duo dans l'opérette de Reynaldo Hahn, voir l'article de Pascal Blanchet dans le présent numéro. 
contenir les huit vers prévus par les auteures. Puisque Germaine Tillion a clairement indiqué que le personnage de Rosine devait chanter, nous avons choisi la deuxième option.

En somme, nous avons voulu adapter la musique au texte de Germaine Tillion en respectant la forme refrain-couplet prévue par les auteures. Nous avons conservé la musique de la section A du duo original pour le refrain et choisi de répéter deux fois la première sous-section de la section $B$ pour le couplet. Parfois, on perçoit une certaine maladresse sur le plan de la prosodie. Nous avons préféré conserver ces maladresses plutôt que d'ajouter ou de supprimer des mots, ou encore d'opérer de nombreux changements de valeurs de notes dans le texte musical. Germaine Tillion a terminé l'écriture de l'air sur un couplet ; cela peut s'expliquer par le fait que le personnage de Marmotte interrompt brusquement Rosine à l'évocation d'un verre d'armagnac.

Exemple musical 1 : Reconstitution de l'air du Verfügbar aux Enfers "Nous avons fait un beau voyage», interprété par Catherine Harrison-Boisvert, enregistré à la Faculté de musique de l'Université de Montréal le 29 juillet 2015; prise de son, Anne-Marie Sylvestre. Écouter.

La version proposée ici diffère radicalement de l'adaptation réalisée par Christophe Maudot et Géraldine Keiflin pour la création du Verfügbar aux Enfers au Théâtre du Châtelet en 2007. Maudot et Keiflin proposent une mise en musique avec orchestre et ont choisi d'accorder la priorité au texte musical des œuvres auxquelles les prisonnières faisaient référence. Ils ont donc adapté le texte à la musique originale et conservé la forme du duo de Ciboulette dans son intégralité. Pour y parvenir, ils ont laissé intacts les numéros 1 et 4 du texte de Germaine Tillion ; ils ont emprunté les quatre derniers vers du numéro 2 pour compléter leur première sous-section de $B$ et les quatre derniers vers du numéro 6 pour compléter leur seconde sous-section de B. Ensuite, ils ont emprunté les refrains 3 et 5 pour en faire les première et seconde sous-sections de $\mathrm{C}$. Enfin, ils ont utilisé le deuxième refrain en guise de section $\mathrm{A}$ conclusive (cette démarche est explicitée dans l'annexe B).

Ainsi, non seulement le texte écrit par Germaine Tillion est-il morcelé, mais il ne figure pas entièrement dans l'adaptation de Maudot et Keiflin. Deux couplets et demi et un refrain - soit 24 vers - sont manquants. À cela, il faut ajouter que le fait que Marmotte interrompe Rosine à propos du verre d'armagnac a perdu son sens. La version proposée ici ne reprend pas la musique originale de Reynaldo Hahn dans son entièreté, mais elle a l'avantage de garder intact le texte des auteures du Verfügbar aux Enfers.

\section{LA PERFORMANCE DE L'AIR DE ROSINE}

Le choix de faire d'un duo virtuose un air pour soliste soulève bien entendu des questions relatives à la praticabilité de l'interprétation. Le tempo adopté par les créateurs de l'opérette est plutôt rapide et la forme en duo permet aux chanteurs de respirer entre les phrases. Toutefois, 11 versions ont été enregistrées entre 1923 
et $1933^{11}$ et les tempos diffèrent d'une version à l'autre. C'est sans compter les interprétations d'amateurs qui pouvaient se procurer dès 1923 la partition des airs de Ciboulette, ainsi que les 569 représentations de l'opérette qui ont eu lieu dans des théâtres de 1923 à $1942^{12}$. Il ne faut ainsi pas exclure le fait que les auteures pouvaient avoir en tête un tempo qui permet au personnage de Rosine de chanter son air seule sans s'essouffler.

Dans une situation réelle de performance de l'air de Rosine, une partition pourrait n'être d'aucun secours, puisque l'actrice l'interprétant ne lirait pas nécessairement la musique. Il a donc été décidé de n'en produire aucune. Idéalement, les actrices prendraient part au processus de reconstruction, écoutant l'œuvre originale et proposant des adaptations au texte. N'ayant accès à aucun instrument de musique, l'actrice pourrait emprunter la tonalité qu'elle désire. Une telle démarche a été empruntée par le Théâtre de la Petite Montagne, une compagnie installée dans le Jura, en France, et proposant surtout des spectacles à but pédagogique. Dans une mise en scène et un décor épurés, deux actrices interprètent les différents personnages à l'aide de marionnettes constituées par des bâtons et des chiffons et chantent les airs sans soutien instrumental ${ }^{13}$.

\section{CONCLUSION}

L'opérette-revue Le Verfügbar aux Enfers est une œuvre fondamentalement musicale et théâtrale. Il faut apporter un soin particulier à la reconstruction des airs, sans qu'ils n'occultent le texte de Germaine Tillion. C'est la raison pour laquelle il nous semble essentiel de privilégier l'adaptation de la musique au texte : 1'approche inverse, consistant à adapter le texte à la musique originale, ne peut qu'altérer le document de Tillion. Cette démarche donnera sans doute un résultat intéressant, mais qui peut difficilement représenter l'opérette-revue écrite par Tillion et ses compatriotes.

Peu importe la démarche adoptée, il est très improbable que le résultat d'un travail de reconstruction corresponde exactement à ce que les auteures avaient en tête au moment d'imaginer leur œuvre. La présente version ne fait pas exception. Mais nous croyons qu'ici, la démarche importe plus que le résultat. Nous avons tenté d'imaginer ce qu'on pouvait retenir de ce duo sans avoir d'instrument de musique sous la main, ni de partition. Nous n'avons pas tant cherché à nous rapprocher de ce que pouvaient faire Tillion et ses codétenues qu'à écarter tout ce qui, à notre avis, leur était impossible à réaliser. Cela dit, deux personnes travaillant de cette même façon peuvent arriver à deux résultats complètement différents ${ }^{14}$. L'important, lorsqu'on cherche à restituer Le Verfügbar aux Enfers de façon à rester fidèle à l'esprit de l'œuvre, c'est de garder en tête que

11 Voir à ce propos la page consacrée à Ciboulette dans l'Encyclopédie multimédia de la comédie musicale théâtrale en France, 1918-1944: http://194.254.96.55/cm/?for=fic\&cleoeuvre=75 (consulté le 10 novembre 2014).

12 Ibid.

13 Pour plus de détails à ce sujet, voir l'article de Cécile Quesney dans le présent numéro.

14 Voir à ce sujet la version proposée dans l'article de Catherine Harrison-Boisvert et Caroline Marcoux-Gendron dans le présent numéro. 
le document de Germaine Tillion, bien que considéré par cette dernière comme un « brouillon ${ }^{15} »$, ne devrait pas être vu comme une simple base que l'on peut modifier à sa guise.

\section{BIBLIOGRAPHIE}

Bauer, Halmuth (1995), Gesichter der Zwangsarbeit. Germaine Tillion über ihre Operette in Ravensbrück, entrevue avec Germaine Tillion, http://gesichter-der-kz-zwangsarbeit.de/die-filme germaine, consulté le 2 novembre 2014.

Encyclopédie multimédia de la comédie musicale théâtrale française 1918-1944, "Ciboulette, opérette en 3 actes et 4 tableaux ", http://194.254.96.55/cm/?for=fic\&cleoeuvre $=75$, consulté le 10 novembre 2014.

Favart, Edmée (2010), La reine de l'opérette. D'Offenbach à Reynaldo Hahn, Malibran CDRG 173.

Georget, Anne (2015), Festins imaginaires, Éditions Montparnasse, 90 min.

Hahn, Reynaldo (1923), « Nous avons fait un beau voyage », tiré de l'opérette Ciboulette, Paris, Éditions Francis Salabert.

Siroka, Jozef (2012), "Retour sur la scène de la douche dans Schindler's List ", Le blogue de Jozef Siroka (15 novembre), http://blogues.lapresse.ca/moncinema/siroka/2012/11/15/retour-sur-la-scenede-la-douche-dans-schindlers-list/, consulté le 8 décembre 2015.

Tillion, Germaine (2005), Le Verfügbar aux Enfers. Une opérette à Ravensbrück, présentation de Tzevan Todorov et Claire Andrieu, Paris, La Martinière.

Tillion, Germaine (2015), Les combats d'une ethnologue. Entretiens avec Frédéric Mitterrand, édition préfacée et annotée par Christian Bromberger et Tzvetan Todorov, Paris, Éditions de l'École des hautes études en sciences sociales.

Unger, David (2008), Germaine Tillion à Ravensbrück, Cinétévé/Arte France, nº 1, 58 min.

15 Voir un entretien accordé par Germaine Tillion à Frédéric Mitterrand et diffusé les 7 et 8 mai 1990 sur Antenne 2 dans le cadre de l'émission Du côté de chez Fred ("Germaine Tillion : Première partie ", 01:18:40). Cet entretien a récemment fait l'objet d'une publication sous forme de livre : voir Tillion 2015, p. 71. 
ANNEXE A : Découpage de l'air "Nous avons fait un beau voyage ", tiré de l'opérette Ciboulette (1923), de Reynaldo Hahn ${ }^{16}$.

- $\quad$ Section A : mes. 7-27 (exemple musical : mes. 7-11);

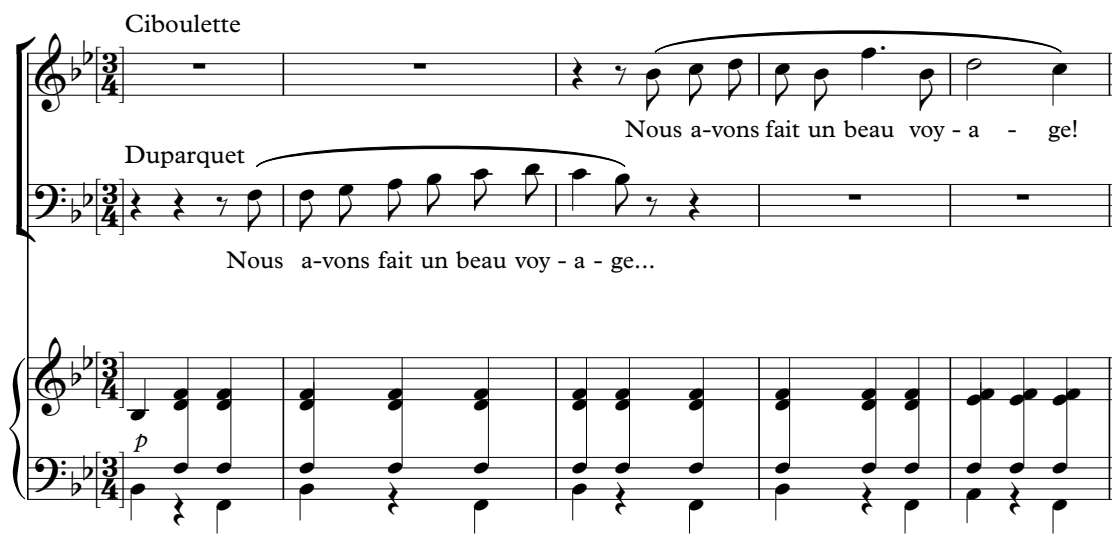

- $\quad$ Section B-1 : mes. 28-35 (exemple musical : mes. 28-31) ;

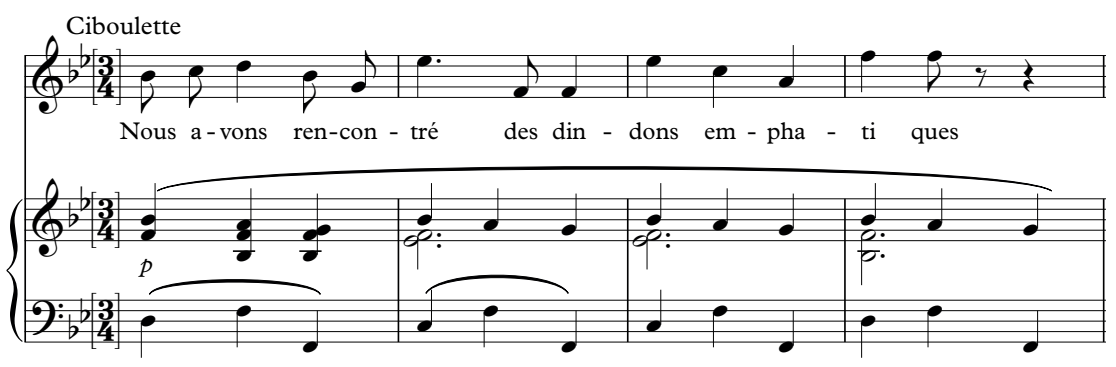

- $\quad$ Section B-2 : mes. 36-43 (exemple musical : mes. 36-39);

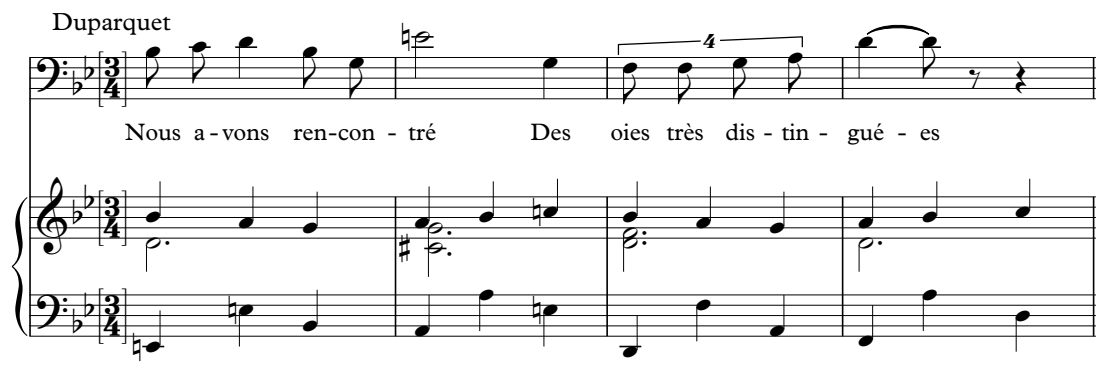

16 Sous licence Domaine public au Canada, la partition est disponible sur le site de l'IMSLP Bibliothèque musicale Petrucci. 
- Section B-3 : mes. 44-51 (exemple musical : mes. 43-47);

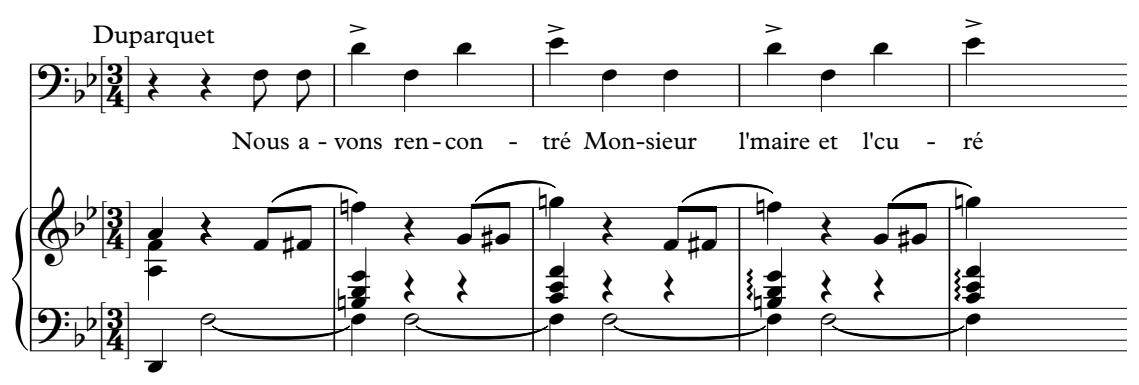

- Section C : mes. 52-67 (exemple musical : mes. 51-55);

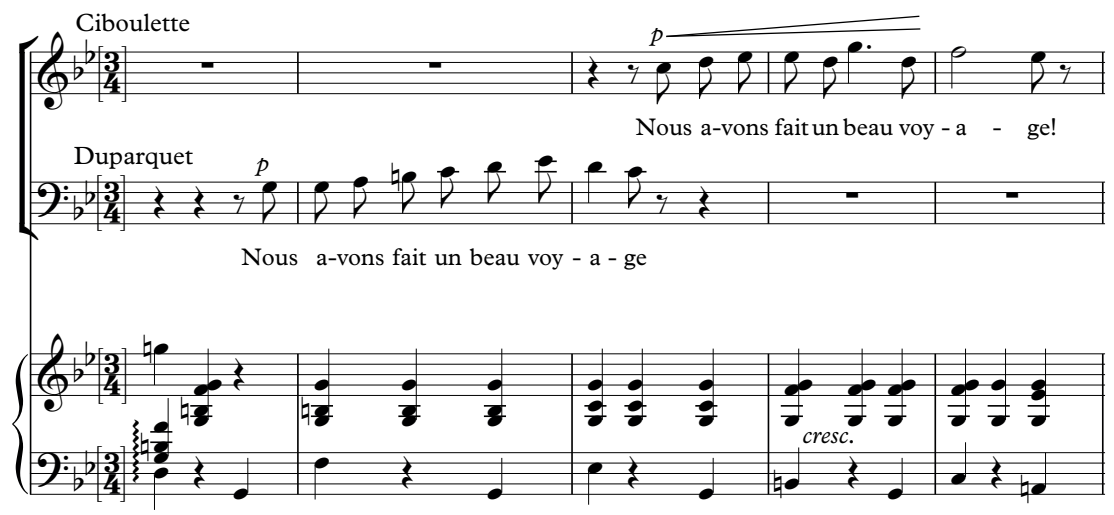

- Section A : mes. 68-87 (exemple musical : mes. 67-71);

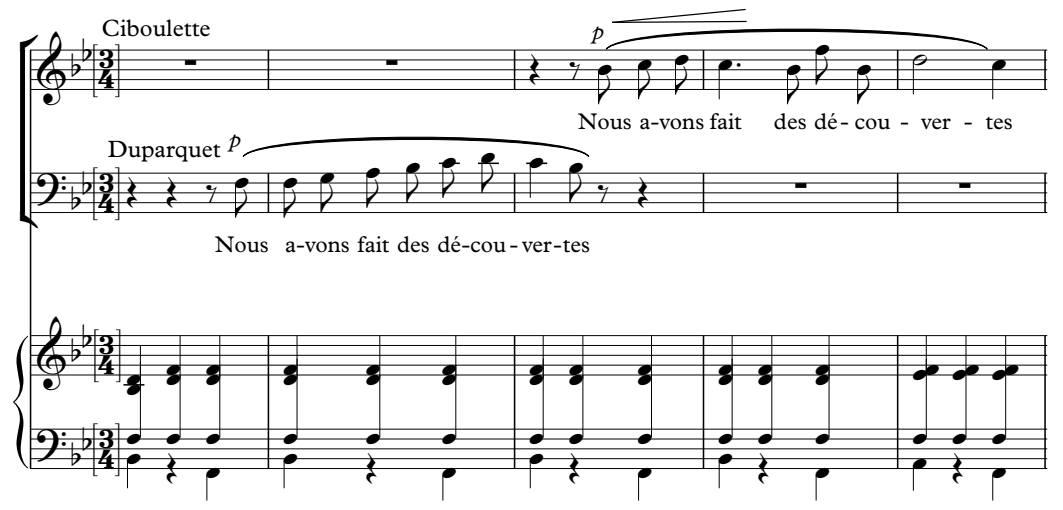


- Section B-1 : mes. 88-95 (exemple musical : mes. 88-91);

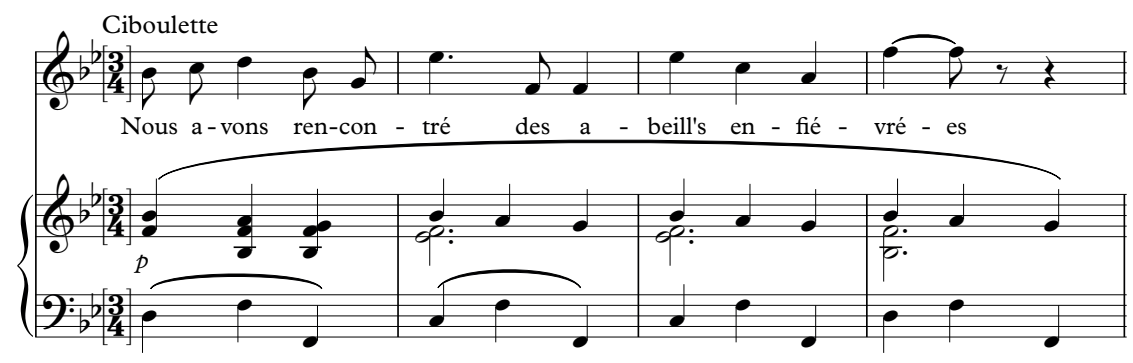

- Section B-2 : mes. 96-103 (exemple musical : mes. 96-99);

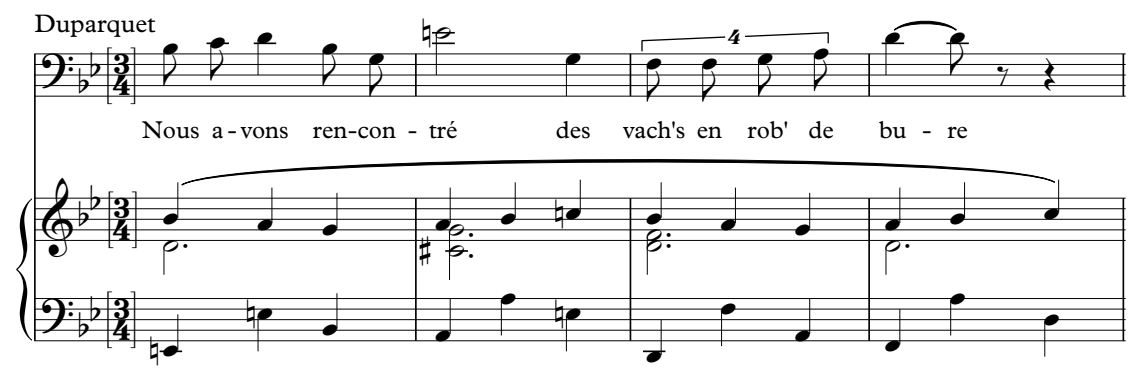

- Section B-3 : mes. 104-111 (exemple musical : mes. 103-107);

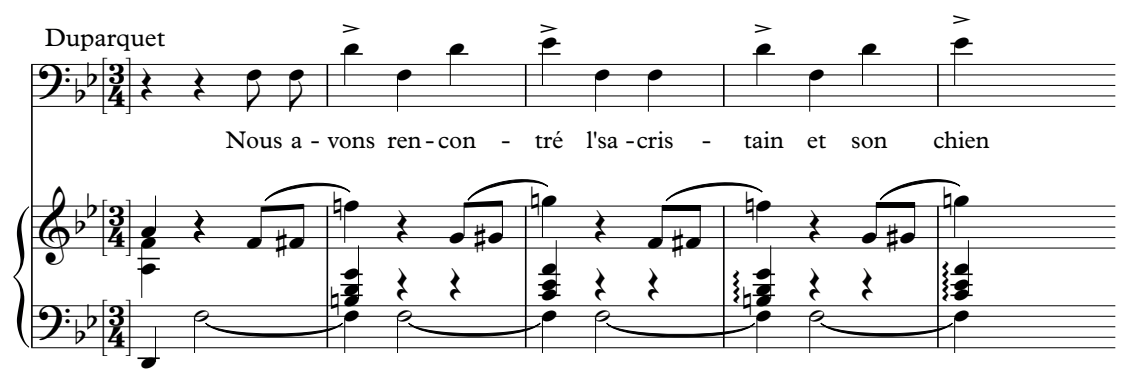

- Section C : mes. 112-127 (exemple musical : mes. 111-115);

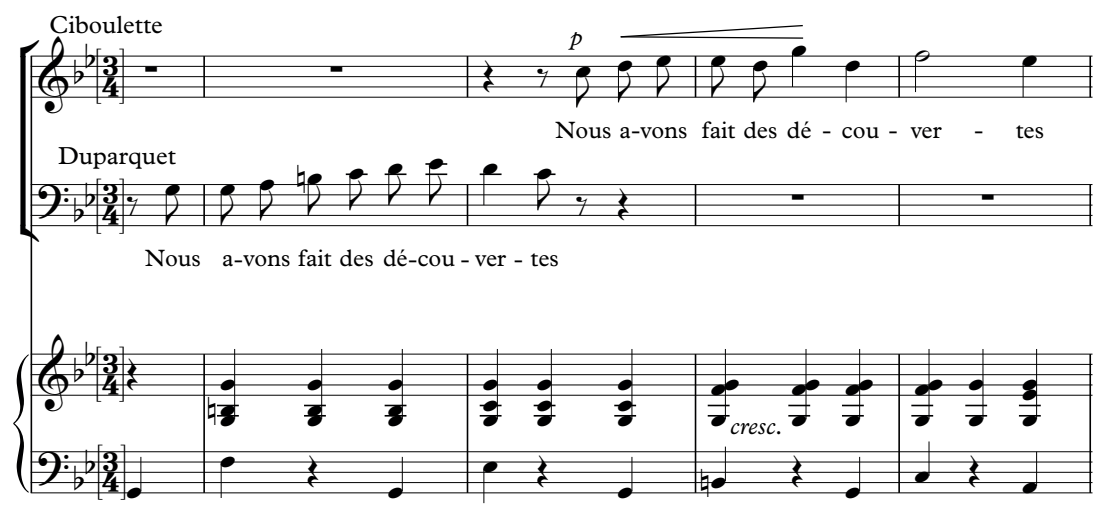


- Section A : mes. 127-155 (exemple musical : mes. 126-130).

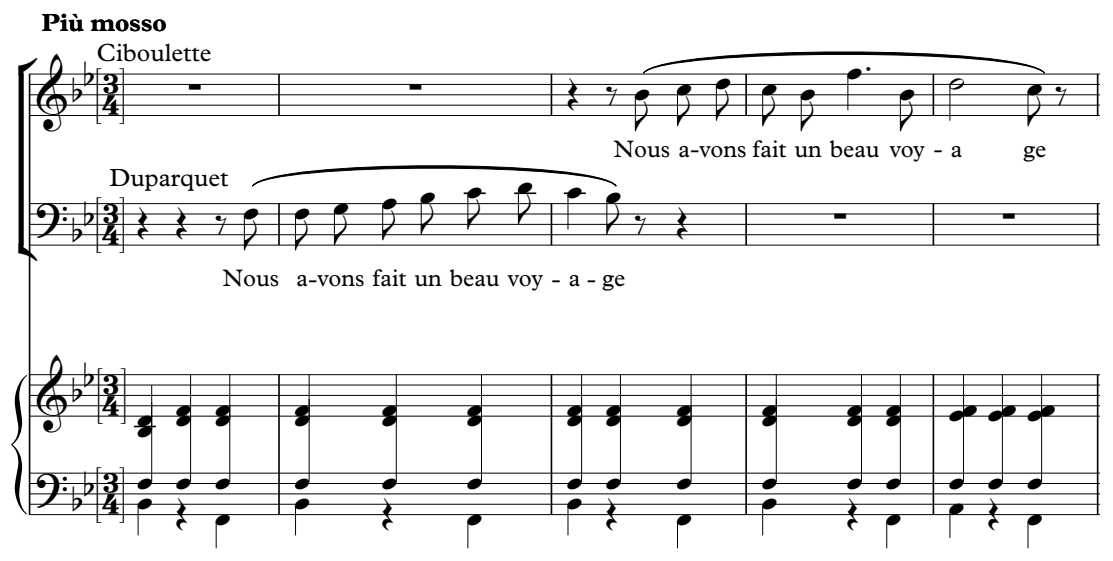


ANNEXE B : Transcription de l'air "Nous avons fait un beau voyage", arrangé par Christophe Maudot et Géraldine Keiflin. Les mots en caractères gras n'apparaissent pas dans le texte du Verfügbar aux Enfers, et ont été ajoutés dans la présente adaptation.

SECTION A

Nous avons fait un beau voyage

Nous arrêtant à tous les pas

Un tuyau comme tout bagage

Emprunté au refrain $n^{\circ} 2$ et correspond à la version originale

Toujours vers l'ouest, nous voguons...

\section{SECTION B}

Nous avons dégusté

Du beurre et du pâté

De la crème en Normandie,

Et du fromage de Brie...

À Riec, savourons

Coquilles et belons,

Bénissant Mélanie

Et sa table bien garnie...

Nous mangeons avec joie

Le foie gras strasbourgeois

Et celui d'Aquitaine,

Et la quiche lorraine...

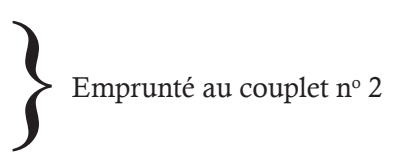

SECTION C

Nous avons fait un beau voyage

Dans tous les jolis coins de France...

Le sourire sur tous les visages

Faisant joyeusement bonne chère...

SECTION A

Nous avons fait un beau voyage

Batifolant à travers prés

Nous abritant sous les ombrages

Rêvant de l'aube à la vesprée...

\section{SECTION B}

Avec du romanée,

Nous avons déjeuné

De potée bourguignonne,

De jambon de Bayonne,

De gratin dauphinois

Et de mets bressois,

De canards rouennais,

De pruneaux agenais.

Nous avons comparé,

Sans pouvoir prononcer

L'eau-de-vie de Cognac

Et puis celle d'Armagnac...

\section{SECTION C}

Nous avons fait un beau voyage

Nous asseyant au bord de l'eau

Écoutant sous le vert feuillage,

Le vent bruire dans les roseaux.

\section{SECTION A ET FINALE}

Nous avons fait un beau voyage,

Nous arrêtant à tous les pas,

Et goûtant dans chaque village,

De bons vins et de bons repas

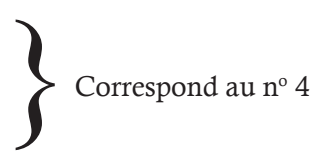

Emprunté au refrain $n^{\circ} 3$
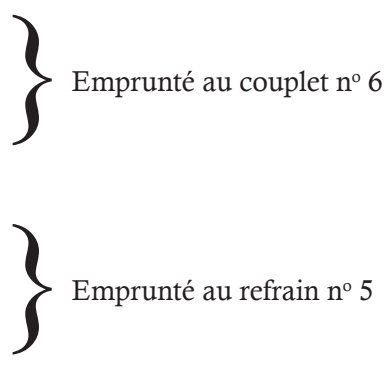

$\int$ Emprunté au refrain $n^{\circ} 2$ 
RECONSTRUIRE LES NUMÉROS CHANTÉS DU VERFÜGBAR AUX ENFERS.

113

LE CAS DE L'AIR DE ROSINE

AnNeXe C : Air de Rosine, manuscrit du Verfügbar aux Enfers (Tillion 2005, p. 154-161).

Rosins: - justement on y a fois gout, Marmothe: - st apies?

hisins (choutrant):

1. Nous as ons fait un bean voy ags, Didkignant autsos of wagnons, un tuyaus cornms toub bagegs, Toufours vers $C^{\prime}$ onut, nous voguons -.

Nows avoms dégushi

Du busre er du fatri,

D' Re crimen en Arrmendie,

Et des formang un Bric..

A Rier, savousons

coppuilles at bel rus

Gérissoni Mèanie

Es sa tablf́' bien gansie -

2. Nous aw ores fait un bean voyagr,

Vous arriboust a vous as pos,

2. poutrant, doms hafus villages,

De bous vins et de lornt refas ..

* A ir en ciboul cte.

Revue musicale OICRM, volume $3, n^{\circ} 2$ 
II 18

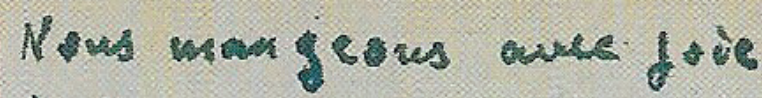

du foir gias strativerguors

Et celus d' tiquirain,

Puis le quich lescouru $\Rightarrow$

Su ba cothe atfentiquer,

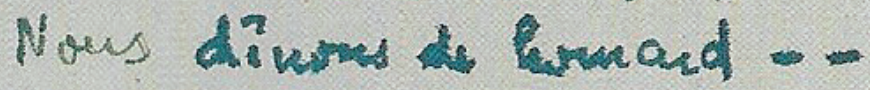

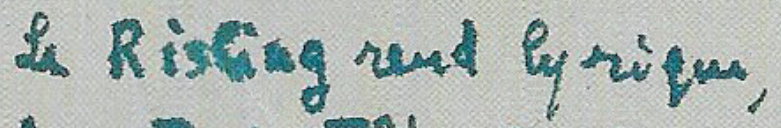

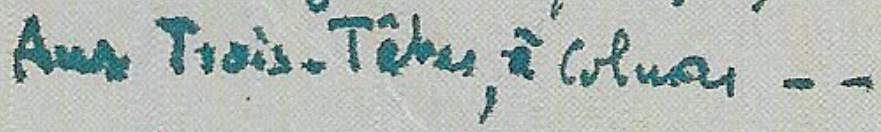

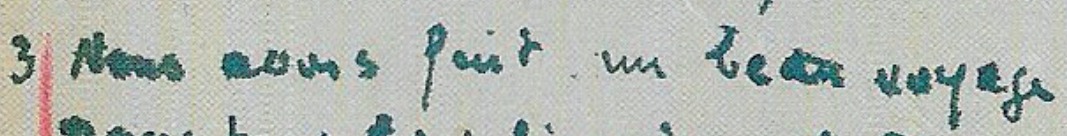

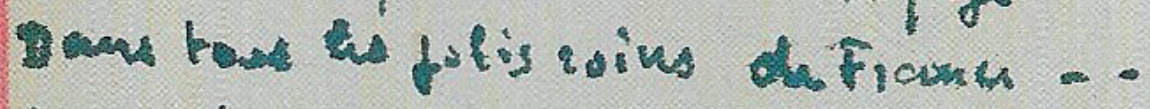

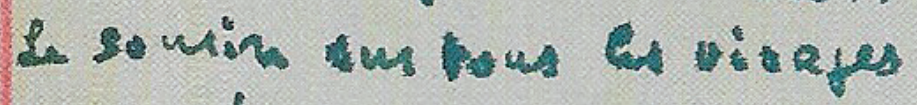

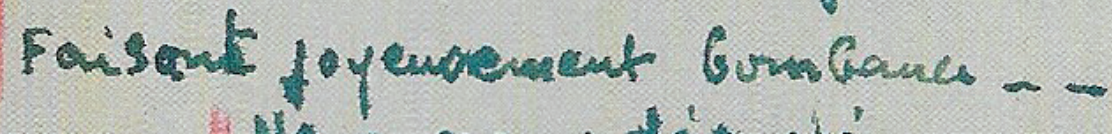

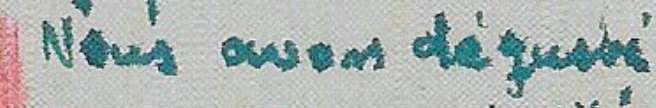

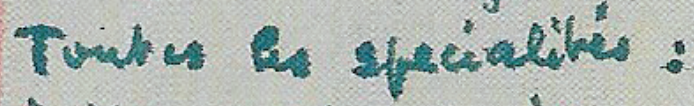

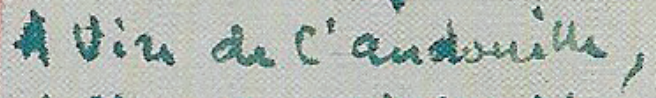

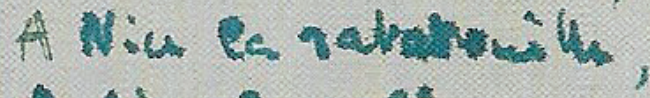

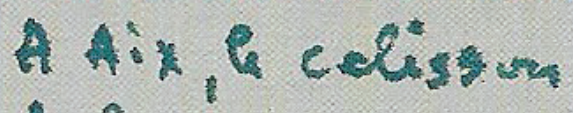

A) Lury, \& squesison,

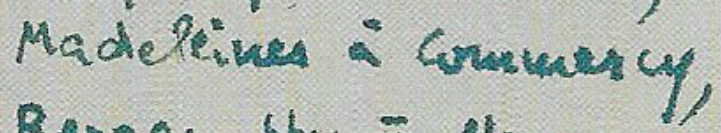

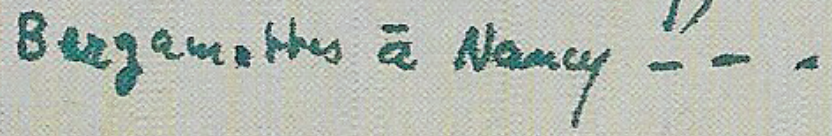


RECONSTRUIRE LES NUMÉROS CHANTÉS DU VERFÜGBAR AUX ENFERS.

115

LE CAS DE L'AIR DE ROSINE

II 19

4 Nons wors pait un bean voyays.. Batigf ant a trewess fies,

Nons abibant sous Ps ombiaces

Revout or l'auhs a la vespré.

Aver dus Romancis

Has arens dé eun

De poter bomgrignone,

Pe jambor of Baydur,

Degration dauphinas

Elo de poulehs bues is

De exuarls ronernais,

D. prukeams acjerais

5 Nous aroms ficil un fean voryages

Nins assugant an Bond di l'en

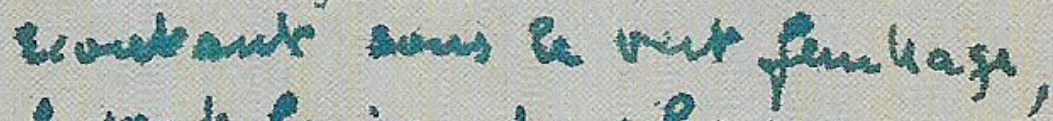

\& vent Crinis doms is rescaus

gintions ches 'A Amise

La tom di fovengal,

A Bar, es enf: twus,

sue'tism, un fribu

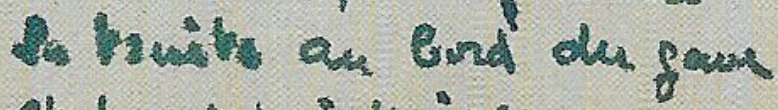
it du mies i Wrigh,

Revue musicale OICRM, volume $3, n^{\circ} 2$ 


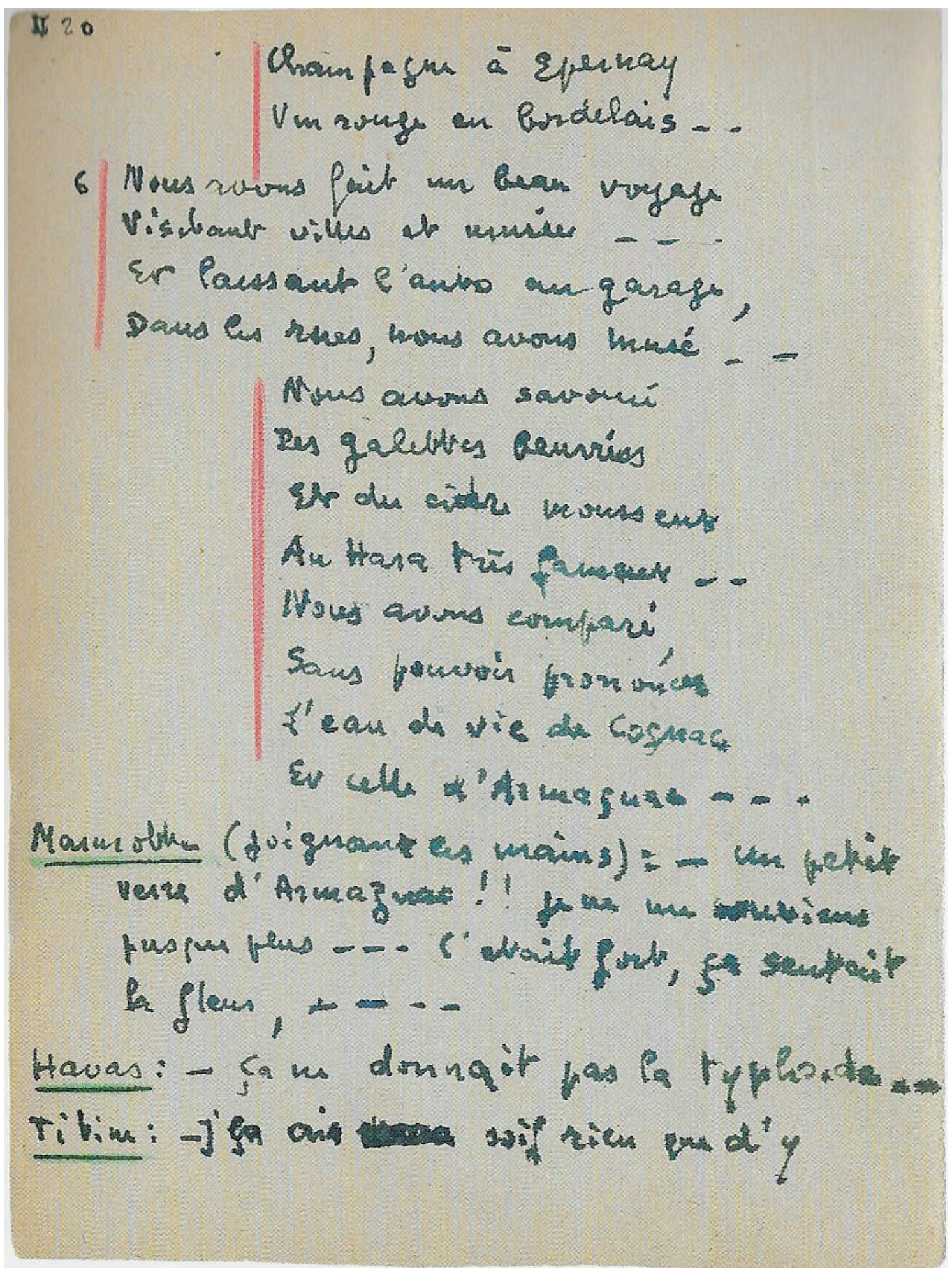

